\section{Rubric Karma}

\author{
Bharti Kalra' Mangesh Tiwaskar²
}

\author{
${ }^{1}$ Department of Obstetrics, Bharti Hospital, Karnal, Haryana, India \\ ${ }^{2}$ Department of Medicine, Karuna Hospital, Mumbai, \\ Maharashtra, India
}

J Soc Health Diab 2019;7:41-42

In Indian philosophy, one's karma (fate or destiny) is thought to be based on actions (and intentions with which those actions were carried out) performed in a previous life or existence. The theory of karma also incorporates the concept of transgenerational or genetic transmission of fate, as parents actions are felt to influence the lives of succeeding generations.

The terminology of karma has been used in metabolic medicine as "metabolic karma" to describe the effects that good metabolic control has on long-term health outcomes. ${ }^{1}$ While metabolic karma was initially thought to be active only within the same individual, recent discussion has highlighted the presence of transgenerational metabolic karma. ${ }^{2}$ This is the name given to the phenomenon of gestational diabetes mellitus, or preexisting diabetes, influencing offspring outcomes in antenatal and later periods of life.

Transgenerational karma has been defined as the influence of preconception, antenatal, and postnatal maternal health on the short-term as well as long-term health of offspring. The term "transgenerational karma" has been postulated to be more comprehensive and inclusive, rather than gluco- or diabetes-centric. Within this broad rubric, we highlight the existence of rubric karma. In Latin, the word rubric means red. Using red or rubric as an allegory for red blood cells and for hemoglobin, rubric karma means the transgenerational impact of maternal hemoglobin status on the health of offspring.

The effect of low or high hemoglobin on maternal health and perinatal outcomes is well documented. Recent research shows that this influence can extend to unborn offspring, and may manifest much later in their lifespan. Hence, a mother's red blood cell health during pregnancy, which is determined (at least in part) by her actions and intentions, is able to influence the health of the next generation. This justifies the moniker "rubric karma." It is also important to note that there is an association of adverse pregnancy outcomes, with both low and high maternal iron levels. ${ }^{3,4}$ These adverse outcomes include gestational diabetes mellitus and other metabolic disorders. Hence, rubric karma can be discussed as an integral part of transgenerational karma.
Address for correspondence Bharti Kalra, MD, DM, Department of Obstetrics and Gynecology, Bharti Hospital, Karnal, 132001, Haryana, India (e-mail: dr.kalrabharti@gmail.com).

From an Indian perspective, however, anemia is a much greater public health problem than iron overload. Inadequate dietary iron, folate, and vitamin B12 intake and bioavailability are major factors which contribute to maternal anemia. These are exacerbated by the increased physiological requirements of iron during pregnancy. Anemia may act by reducing placental transfer of oxygen to fetus, slowing growth and development of placenta, and affecting endocrine gland function.

Maternal anemia leads to uterine growth retardation and preterm or low-birth-weight babies. These offspring usually maintain suboptimal growth patterns in infancy, childhood, and puberty, and end up perpetuating a vicious transgenerational cycle of ill health. ${ }^{5}$ This intergenerational cycle is what we call rubric karma.

Maternal anemia has been found to increase the risk of schizophrenia in offspring. A population-based cohort of 6,872 offspring, born between 1959 and 1967, ${ }^{6}$ followed them up for development of schizophrenia spectrum disorders (SSDs). In the Prenatal Determinants of Schizophrenia (PDS) study, mean maternal hemoglobin of $\leq 10.0 \mathrm{~g} / \mathrm{dL}$ was associated with an adjusted rate ratio of 3.73 (95\% confidence interval [CI]: $1.41-9.81 ; p=0.008)$, SSDs as compared to a hemoglobin $\geq 12.0 \mathrm{~g} / \mathrm{dL}$ and a $27 \%$ decrease in the rate of SSDs was observed for every $1 \mathrm{~g} / \mathrm{dL}$ increase in mean maternal hemoglobin.

In a Danish cohort of 111,572 singleton births (born between 1978 and 1998), members were followed up from their 10th birthday till onset of schizophrenia, death, or December 31, 2008, whichever occurred first. After adjustment, offspring of mothers who had been diagnosed to have anemia during pregnancy had a 1.60 -fold $(95 \% \mathrm{CI}$ : 1.16-2.15) greater risk of schizophrenia.?

In an Australian population-based study, the risk of severe intellectual disability was increased in offspring of women with anemia (odds ratio: 5.26; 95\% CI: 2.16-12.80). This odds ratio was greater than that observed in children of mother with asthma, diabetes, renal or urinary condition, or epilepsy. ${ }^{8}$ This has been postulated to be due to impaired oxygen delivery to the brain, which disrupts neurodevelopment and function, including myelination and dopamine-rich transmission.

\section{received}

April 1, 2019

accepted

April 6, 2019
DOI https://doi.org/

$10.1055 / \mathrm{s}-0039-1692373$

ISSN 2321-0656.
C2019 Novo Nordisk Education Foundation
License terms

() (1) $\odot \circledast$ 
The Pune Maternal Nutrition study measured various metabolic parameters at 18 and 28 weeks of gestation in 700 rural pregnant women, and then assessed their offspring at 6 years of age. Higher maternal erythrocyte foliate concentration at 28 weeks predicted higher offspring adiposity and a higher HOMA-R $(p<0.01)$. Low maternal vitamin B12 on the other hand (18 weeks, $p=0.03$ ) predicted higher HOMA-R in offspring. This study suggested that noniron hematometabolic health of pregnant mothers could influence metabolic health of offspring. ${ }^{9}$

In the Avon Longitudinal study of parents and children, maternal hemoglobin, iron supplementation, and foodbased iron intake were assessed during pregnancy, and analyzed with regard to blood pressure of offspring at 7 years of age. Maternal anemia during third trimester was associated with lower systolic blood pressure in offspring at 7 years of age ( $\beta=1.09 ; 95 \mathrm{CI}: 2.21,0.05 \mathrm{~mm} \mathrm{Hg}) ; p=0.04$ ). The association was stronger in women who did not take iron supplements during antenatal period, but was attenuated after adjustment for cofounders. ${ }^{10}$ The authors postulated that low hemoglobin might reflect greater plasma volume expansion, and better health, in well-nourished populations, rather than indicate iron deficiency.

In the Indian population, therefore, it makes sense to supplement iron and hematopoietic vitamins, in order to achieve and maintain normal hemoglobin levels before and during pregnancy. ${ }^{11} \mathrm{~A}$ simple intervention such as this will help ensure healthy rubric karma for our future generation.

Conflict of Interest

None declared.

\section{References}

1 Thomas MC. Glycemic exposure, glycemic control, and metabolic karma in diabetic complications. Adv Chronic Kidney Dis 2014;21(3):311-317

2 Kalra B, Kalra S, Unnikrishnan AG, Baruah MP, Khandelwal D, Gupta Y. Transgenerational Karma. Indian J Endocrinol Metab 2017;21(2):265-267

3 Scholl TO. Iron status during pregnancy: setting the stage for mother and infant. Am J Clin Nutr 2005;81(5):1218S-1222S

4 Lao TT, Chan PL, Tam KF. Gestational diabetes mellitus in the last trimester - a feature of maternal iron excess? Diabet Med 2001;18(3):218-223

5 Kalaivani K. Prevalence \& consequences of anaemia in pregnancy. Indian J Med Res 2009;130(5):627-633

6 Insel BJ, Schaefer CA, McKeague IW, Susser ES, Brown AS. Maternal iron deficiency and the risk of schizophrenia in offspring. Arch Gen Psychiatry 2008;65(10):1136-1144

7 Sørensen HJ, Nielsen PR, Pedersen CB, Mortensen PB. Association between prepartum maternal iron deficiency and offspring risk of schizophrenia: population-based cohort study with linkage of Danish national registers. Schizophr Bull 2011;37(5):982-987

8 Leonard H, de Klerk N, Bourke J, Bower C. Maternal health in pregnancy and intellectual disability in the offspring: a population-based study. Ann Epidemiol 2006;16(6):448-454

9 Yajnik CS, Deshpande SS, Jackson AA, et al. Vitamin B12 and folate concentrations during pregnancy and insulin resistance in the offspring: the Pune Maternal Nutrition Study. Diabetologia 2008;51(1):29-38

10 Brion MJ, Leary SD, Smith GD, McArdle HJ, Ness AR. Maternal anemia, iron intake in pregnancy, and offspring blood pressure in the Avon Longitudinal Study of Parents and Children. Am J Clin Nutr 2008;88(4):1126-1133

11 Allen LH. Anemia and iron deficiency: effects on pregnancy outcome. Am J Clin Nutr 2000;71(5, Suppl):1280S-1284S 